\title{
THE FRACTIONAL CHROMATIC NUMBER OF THE DIRECT PRODUCT OF GRAPHS
}

\author{
XUDING ZHU* \\ Department of Applied Mathematics, National Sun Yat-sen University, Kaohsiung, 814-0180, \\ Taiwan 80424
}

(Received 2 May, 2000; accepted 16 February 2001)

\begin{abstract}
This paper discusses the fractional chromatic number of the direct product of graphs. It is proved that if $H$ is a circulant graph $G_{d}^{k}$, or a Kneser graph, or a direct sum of such graphs, then for any graph $G, \chi_{f}(G \times H)=\min \left\{\chi_{f}(G), \chi_{f}(H)\right\}$.
\end{abstract}

2000 Mathematics Subject Classification. 05C15.

1. Introduction. All graphs considered in this paper are finite. For graphs $G=(V, E)$ and $H=\left(V^{\prime}, E^{\prime}\right)$, the direct product (or the categorical product) $G \times H$ has vertex set $V \times V^{\prime}$ and in which $\left(x, x^{\prime}\right)$ is adjacent to $\left(y, y^{\prime}\right)$ if and only if $x y \in E$ and $x^{\prime} y^{\prime} \in E^{\prime}$.

Suppose $G=(V, E)$ is a graph. Let $\mathcal{I}$ denote the family of independent sets of $G$. A mapping $f: \mathcal{I} \rightarrow[0,1]$ is called an $r$-fractional colouring of $G$ if $\sum_{X \in \mathcal{I}} f(X) \leq r$ and for each $v \in V(G), \sum_{v \in X, X \in \mathcal{I}} f(X) \geq 1$. The fractional chromatic number $\chi_{f}(G)$ of $G$ is the least $r$ for which $G$ has an $r$-fractional colouring.

This paper investigates the fractional chromatic number of the direct product of graphs. A homomorphism from $G$ to $H$ is a mapping $h: V(G) \rightarrow V(H)$ which preserves edges, i.e., if $x y \in E(G)$ then $h(x) h(y) \in E(H)$. We say $G$ is homomorphic to $H$ if there exists a homomorphism from $G$ to $H$. It is easy to see (and well-known) that if $G$ is homomorphic to $H$ then $\chi_{f}(G) \leq \chi_{f}(H)$. Since $G \times H$ is homomorphic to $G$ and $H$ (the projections are homomorphisms), it follows that $\chi_{f}(G \times H) \leq$ $\min \left\{\chi_{f}(G), \chi_{f}(H)\right\}$. An interesting question (asked by Perles independently [8]) is whether or not the equality holds for all graphs $G$ and $H$.

QUESTION 1.1. Is it true that for any graphs $G$ and $H$, we have

$$
\chi_{f}(G \times H)=\min \left\{\chi_{f}(G), \chi_{f}(H)\right\} ?
$$

A fractional clique of $G$ is a mapping $\sigma: V(G) \rightarrow[0,1]$ such that for each independent set $X$ of $G, \sum_{v \in X} \sigma(v) \leq 1$. For any subset $X$ of $V(G)$, let $\sigma(X)=\sum_{v \in X} \sigma(v)$. The weight $w(\sigma)$ of a fractional clique $\sigma$ of $G$ is defined as $w(\sigma)=\sigma(V(G))$. The fractional clique number $\omega_{f}(G)$ of $G$ is the maximum weight of a fractional clique of $G$. It is well-known [9] that, for any graph $G, \chi_{f}(G)$ is calculated by solving a linear programming problem and $\omega_{f}(G)$ is calculated by solving

*Supported in part by the National Science Council of R.O.C. under grant NSC88-2115-M-110-001. 
the dual of that linear programming problem. Hence $\omega_{f}(G)=\chi_{f}(G)$. Therefore, (1) is equivalent to

$$
\omega_{f}(G \times H)=\min \left\{\omega_{f}(G), \omega_{f}(H)\right\}
$$

Suppose $g$ and $h$ are fractional cliques of $G$ and $H$, respectively. Let $\phi: V(G \times H) \rightarrow[0,1]$ be defined by $\phi(x, y)=g(x) h(y) / q$, where $q=\max \left\{\omega_{f}(G)\right.$, $\left.\omega_{f}(H)\right\}$. It seems natural to wonder if $\phi$ is a fractional clique of $G \times H$. This leads to the following question.

Question 1.2. Suppose $g$, $h$ are fractional cliques of $G$ and $H$, respectively. Let $U$ be an independent set of $G \times H$. Is it true that

$$
\sum_{(x, y) \in U} g(x) h(y) \leq \max \left\{\omega_{f}(G), \omega_{f}(H)\right\} ?
$$

The "integral version" of the fractional chromatic number and the fractional clique number are two different parameters: the chromatic number and the clique number. The integral version of Equations (1) and (1') then become two equations. It is interesting to note that these two equations are of very different nature. It is trivial that for all graphs $G, H$,

$$
\omega(G \times H)=\min \{\omega(G), \omega(H)\} .
$$

However, it is unknown whether or not

$$
\chi(G \times H)=\min \{\chi(G), \chi(H)\}
$$

for all graphs $G, H$. It was conjectured by Hedetniemi [5] that (3) holds for all graphs. This conjecture has attracted considerable attention, and yet only some very special cases of the conjecture have been verified $[\mathbf{1 , 4 , 1 1 , 1 5}$. It seems that (1) is sandwiched between (2) and (3). However, it is unknown if there are any intrinsic connection between these equalities. The main result of this paper is the following theorem.

THEOREM 1.3. If $H$ is a circulant graph $G_{d}^{k}$, or a Kneser graph, or a direct sum of such graphs, then for any graph $G, \chi_{f}(G \times H)=\min \left\{\chi_{f}(G), \chi_{f}(H)\right\}$.

Theorem 1.3 generalizes some earlier results concerning the independence number of the direct product of graphs [3,7]. A result of Frank1 [3], which determines the independence number of the direct product of Kneser graphs, is equivalent to saying that if $G$ and $H$ are both Kneser graphs then $\chi_{f}(G \times H)=\min \left\{\chi_{f}(G), \chi_{f}(H)\right\}$. A simple proof of Frankl's result is given by Kirsch [8], by studying Question 1.1. It is known [15] that if $\chi(H) \geq n$ then $\chi\left(K_{n} \times H\right)=n$. Therefore, it follows from Theorem 1.3 that $\chi_{f}\left(K_{n} \times H\right)=\chi\left(K_{n} \times H\right)$ if $\chi_{f}(H) \geq n$. This provides a simple construction of graphs with their fractional chromatic number equal to their chromatic number.

2. Relation to Hedetniemi's conjecture. It is unknown if there is any intrinsic connection between (1) and Hedetniemi's conjecture. The following lemma shows that if (1) holds for $G$ and $H$, then (3) holds for some related graphs. 
Suppose $G=(V, E)$ and $G^{\prime}=\left(V^{\prime}, E^{\prime}\right)$ are graphs. The lexicographic product $G\left[G^{\prime}\right]$ has vertex set $V \times V^{\prime}$, and has $\left(x, x^{\prime}\right) \sim\left(y, y^{\prime}\right)$ if and only if either $x \sim y$ or $x=y$ and $x^{\prime} \sim y^{\prime}$.

Lemma 2.1. If $\chi_{f}(G \times H)=\min \left\{\chi_{f}(G), \chi_{f}(H)\right\}$, then there exists an integer $n$ such that $\chi\left(G\left[K_{n}\right] \times H\left[K_{n}\right]\right)=\min \left\{\chi\left(G\left[K_{n}\right]\right), \chi\left(H\left[K_{n}\right]\right)\right\}$.

Proof. It suffices to show that there is an integer $n$ such that

$$
\chi\left(G\left[K_{n}\right] \times H\left[K_{n}\right]\right) \geq \min \left\{\chi\left(G\left[K_{n}\right], \chi\left(H\left[K_{n}\right]\right)\right\} .\right.
$$

It is well-known [9] for any graph $G^{\prime}$ there is an integer $k$ such that for any positive integer $l, \chi\left(G^{\prime}\left[K_{l k}\right]\right)=l k \chi_{f}\left(G^{\prime}\right)$. Therefore there exists an integer $n$ such that $\chi\left(G\left[K_{n}\right]\right)=n \chi_{f}(G), \chi\left(H\left[K_{n}\right]\right)=n \chi_{f}(H)$ and $\chi\left((G \times H)\left[K_{n}\right]\right)=n \chi_{f}(G \times H)$.

The vertices of $(G \times H)\left[K_{n}\right]$ are triples $(g, h, i)$, where $g \in V(G), h \in V(H)$, $i \in V\left(K_{n}\right)$. Two vertices $(g, h, i)$ and $\left(g^{\prime}, h^{\prime}, i^{\prime}\right)$ are adjacent if and only if either $g \sim g^{\prime}$, $h \sim h^{\prime}$ or $(g, h)=\left(g^{\prime}, h^{\prime}\right)$ and $i \neq i^{\prime}$. The vertices of $G\left[K_{n}\right] \times H\left[K_{n}\right]$ are 4-tuples $(g, i, h, j)$, where $g \in V(G), h \in V(H), i, j \in V\left(K_{n}\right)$. Two vertices $(g, i, h, j)$ and $\left(g^{\prime}, i^{\prime}, h^{\prime}, j^{\prime}\right)$ are adjacent if and only if one of the following is true:

- $g \sim g^{\prime}$ and $h \sim h^{\prime}$

- $(g, h)=\left(g^{\prime}, h^{\prime}\right)$ and $i \neq i^{\prime}$ and $j \neq j^{\prime}$;

- $g=g^{\prime}, i \neq i^{\prime}$ and $h \sim h^{\prime}$;

- $g \sim g^{\prime}, h=h^{\prime}$ and $j \neq j^{\prime}$.

It is straightforward to verify that the mapping $f(g, h, i)=(g, i, h, i)$ is a homomorphism from $(G \times H)\left[K_{n}\right]$ to $G\left[K_{n}\right] \times H\left[K_{n}\right]$. Therefore $\chi\left((G \times H)\left[K_{n}\right]\right) \leq$ $\chi\left(G\left[K_{n}\right] \times H\left[K_{n}\right]\right)$. As

$$
\begin{aligned}
\chi\left((G \times H)\left[K_{n}\right]\right) & =n \chi_{f}(G \times H) \\
& =n \min \left\{\chi_{f}(G), \chi_{f}(H)\right\} \\
& =\min \left\{\chi\left(G\left[K_{n}\right]\right), \chi\left(H\left[K_{n}\right]\right)\right\},
\end{aligned}
$$

we conclude that

$$
\chi\left(G\left[K_{n}\right] \times H\left[K_{n}\right]\right) \geq \min \left\{\chi\left(G\left[K_{n}\right]\right), \chi\left(H\left[K_{n}\right]\right)\right\} .
$$

In view of Theorem 1.3, Lemma 2.1 proves some new cases of Hedetniemi's conjecture. For example, if $G=K(a n, a k)\left[K_{m}\right], H=K(b n, b k)\left[K_{m}\right]$, where $m=$ $\left(a^{2} b+a b^{2}\right) k$, then $\chi(G \times H)=\chi(G)=\chi(H)$. $(K(k, d)$ denotes a Kneser graph; see definition in Section 5). Such instances are not included in other proven special cases of Hedetniemi's conjecture.

3. Fractional persistent graphs. Suppose $U$ is an independent set of $G \times H$, and that $g, h$ are fractional cliques of $G, H$ respectively. We define the mapping $\phi_{g, h}$ as follows:

$$
\phi_{g, h}(U)=\sum_{(x, y) \in U} g(x) h(y)
$$


As an attempt to prove that Equation 1 holds for all graphs $G$ and $H$, we introduce the concept of a fractional persistent graph as follows.

Definition 3.1. Suppose $h$ is a fractional clique of $H$. We say $h$ is a persistent fractional clique of $H$ if for any graph $G$, for any fractional clique $g$ of $G$, and for any independent set $U$ of $G \times H$, we have

$$
\phi_{g, h}(U) \leq \max \left\{\omega_{f}(G), \omega_{f}(H)\right\}
$$

Definition 3.2. We say a graph $H$ is fractional persistent if $H$ has a persistent fractional clique $h$ of weight $\omega_{f}(H)$. We say a graph $H$ is strongly fractional persistent if every fractional clique of $H$ is persistent.

By definition, a strongly fractional persistent graph is fractional persistent.

Leмma 3.1. If $H$ is fractional persistent then, for any graph $G$,

$$
\chi_{f}(G \times H)=\min \left\{\chi_{f}(G), \chi_{f}(H)\right\} .
$$

Proof. Let $g$ be a fractional clique of $G$ of weight $\omega_{f}(G)$, and $h$ be a persistent fractional clique of $H$ of weight $\omega_{f}(H)$. Suppose $q=\max \left\{\omega_{f}(G), \omega_{f}(H)\right\}$. Let $f: V(G \times H) \rightarrow[0,1]$ be defined as

$$
f(x, y)=\frac{g(x) h(y)}{q} .
$$

Since $h$ is persistent, for any independent set $U$ of $G \times H, f(U) \leq 1$. Hence $f$ is a fractional clique of $G \times H$. It is straightforward to verify that the weight of $f$ is $\min \left\{\omega_{f}(G), \omega_{f}(H)\right\}$. Therefore

$$
\omega_{f}(G \times H) \geq \min \left\{\omega_{f}(G), \omega_{f}(H)\right\} .
$$

Question 1.2 is equivalent to ask whether or not every graph is strongly fractional persistent. A positive answer to the following (weaker) question would imply a positive answer to Question 1.1.

QUESTION 3.2. Is it true that every graph $H$ is fractional persistent?

The remaining part of this paper gives some sufficient conditions for a graph to be fractional persistent and strongly fractional persistent.

Lemma 3.3. Suppose $H$ is fractional persistent. If $H$ admits a homomorphism to $H^{\prime}$ and $\omega_{f}\left(H^{\prime}\right)=\omega_{f}(H)$ then $H^{\prime}$ is also fractional persistent.

Proof. Let $h$ be a persistent fractional clique of $H$, let $\phi$ be a homomorphism from $H$ to $H^{\prime}$. For each $x^{\prime} \in V\left(H^{\prime}\right)$, let $h^{\prime}\left(x^{\prime}\right)=\sum_{x \in \phi^{-1}\left(x^{\prime}\right)} h(x)$ (if $\phi^{-1}\left(x^{\prime}\right)=\emptyset$ then $\left.h^{\prime}\left(x^{\prime}\right)=0\right)$. Then $h^{\prime}$ is a fractional clique of $H^{\prime}$ of weight $\omega_{f}\left(H^{\prime}\right)=\omega_{f}(H)$. Let $G$ be any graph, $g$ be any fractional clique of $G$, and $U^{\prime}$ be any independent set of $G \times H^{\prime}$. Let $U=\left\{(x, y) \in V(G \times H):(x, \phi(y)) \in U^{\prime}\right\}$. It is easy to verify that $U$ is an 
independent set of $G \times H$. As $h$ is a persistent fractional clique of $H$, we have $\phi_{g, h}(U) \leq \max \left\{\omega_{f}(G), \omega_{f}(H)\right\}$. It follows from the definitions that $\phi_{g, h^{\prime}}\left(U^{\prime}\right)=$ $\phi_{g, h}(U)$. Therefore $\phi_{g, h^{\prime}}\left(U^{\prime}\right) \leq \max \left\{\omega_{f}(G), \omega_{f}\left(H^{\prime}\right)\right\}$. Hence $h^{\prime}$ is a persistent fractional clique of $H^{\prime}$ and $H^{\prime}$ is fractional persistent.

Our next result shows that one can construct new strongly fractional persistent graphs from old ones.

Suppose $H_{1}=\left(V_{1}, E_{1}\right)$ and $H_{2}=\left(V_{2}, E_{2}\right)$ are two vertex disjoint graphs. The direct sum $H_{1}+H_{2}$ has vertex set $V_{1} \cup V_{2}$ and edge set $E_{1} \cup E_{2} \cup\left\{x y: x \in V_{1}\right.$, $\left.y \in V_{2}\right\}$. It is obvious that $\omega_{f}\left(H_{1}+H_{2}\right)=\omega_{f}\left(H_{1}\right)+\omega_{f}\left(H_{2}\right)$.

THEOREM 3.4. If $H_{1}=\left(V_{1}, E_{1}\right)$ and $H_{2}=\left(V_{2}, E_{2}\right)$ are strongly fractional persistent then the direct sum $\mathrm{H}_{1}+\mathrm{H}_{2}$ is also strongly fractional persistent.

Proof. Let $G=(V, E)$ be an arbitrary graph. Suppose $g$ is a fractional clique of $G$, and $h$ is a fractional clique of $H_{1}+H_{2}$. Let $q=\max \left\{\omega_{f}(G), \omega_{f}\left(H_{1}+H_{2}\right)\right\}$.

Let $U$ be an independent set of $G \times\left(H_{1}+H_{2}\right)$. We need to show that

$$
\phi_{g, h}(U)=\sum_{(x, y) \in U} g(x) h(y) \leq q .
$$

For each vertex $x$ of $G$, let $U_{x}=\left\{y \in V_{1} \cup V_{2}:(x, y) \in U\right\}$. For $i=1,2$, let $A_{i}=\left\{x \in V: U_{x} \cap V_{i} \neq \emptyset\right\}$. It follows from the definition that if $x \in A_{1}, x^{\prime} \in A_{2}$ then $x \nsim x^{\prime}$. Let $B=A_{1} \cap A_{2}, B_{i}=A_{i}-B$. Then $B$ is an independent set of $G$, and $\left(B_{1} \cup B_{2}\right) \cap N_{G}[B]=\emptyset, N_{G}\left(B_{1}\right) \cap B_{2}=\emptyset$. Here $N_{G}(X)$ (or $N(X)$ if there is no confusion) is the neighbour set of $X$, i.e., $N(x)=\{y: \exists x \in X, x \sim y\}$, and $N_{G}[X]=$ $N_{G}(X) \cup X$ (or $N[X]$ if there is no confusion) is the closed neighbour set of $X$. With an abuse of notation, we also let $B_{i}$ stand for the subgraph of $G$ induced by $B_{i}$.

Let $b=g(B)$ and for $i=1,2$, let $a_{i}=\max \left\{g(X): X\right.$ is an independent set of $\left.B_{i}\right\}$. Suppose $a_{i}=g\left(X_{i}\right)$, where $X_{i}$ is an independent set of $B_{i}$. Then $X_{1} \cup X_{2} \cup B$ is an independent set of $G$. Therefore $a_{1}+a_{2}+b \leq 1$.

For $i=1,2$, let $\phi_{i}: B_{i} \rightarrow[0,1]$ be defined as $\phi(x)=g(x) / a_{i}$. Then $\phi_{i}$ is a fractional clique of $B_{i}$. Since $U \cap\left(B_{i} \times\left(H_{1}+H_{2}\right)\right)=U \cap\left(B_{i} \times H_{i}\right)$ is an independent set of $B_{i} \times H_{i}$, and since $H_{i}$ is fractional persistent, we conclude that

$$
\sum_{(x, y) \in U \cap\left(B_{i} \times H_{i}\right)} \phi_{i}(x) h(y) \leq \max \left\{\chi_{f}\left(B_{i}\right), \chi_{f}\left(H_{i}\right)\right\} .
$$

Therefore

$$
\sum_{(x, y) \in U \cap\left(B_{i} \times H_{i}\right)} g(x) h(y) \leq a_{i} \max \left\{\chi_{f}\left(B_{i}\right), \chi_{f}\left(H_{i}\right)\right\} \leq a_{i} q .
$$

(Note that $\omega_{f}\left(B_{i}\right) \leq \omega_{f}(G) \leq q$.)

As

$$
\sum_{(x, y) \in U \cap\left(B \times\left(H_{1}+H_{2}\right)\right)} f(x) h(y) \leq f(B) \omega_{f}\left(H_{1}+H_{2}\right) \leq b q,
$$

we conclude that 


$$
\phi_{g, h}(U)=\sum_{(x, y) \in U} g(x) h(y) \leq b q+a_{1} q+a_{2} q \leq q .
$$

This completes the proof of Theorem 3.4.

COROLlaRY 3.5. If $H_{i}$ is strongly fractional persistent for $i=1,2, \cdots, n$, then $H_{1}+H_{2}+\cdots+H_{n}$ is strongly fractional persistent.

Since $K_{1}$ is obviously fractional persistent, we have

COROLLARY 3.6. The complete graphs are strongly fractional persistent.

4. Localized fractional clique. To prove that a graph $H$ is strongly fractional persistent amounts to proving that every fractional clique of $H$ is persistent. It follows from Corollary 3.6 that if the total weight of a fractional clique $h$ is assigned to a clique of $H$, then $h$ is persistent. To be precise, we have the following lemma.

Lemma 4.1. Suppose $h$ is a fractional clique of $H$ of weight $r$. If for all $x$ with $h(x)>0$ we have $f(N(x))=r-f(x)$, then $f$ is a persistent fractional clique.

Definition 4.1. A fractional clique $h$ of $H$ of weight $r$ is called a localized fractional clique if for all $X \subseteq V(H)$ with $h(X)>0$, we have $h(N(X)) \geq$ $\min \{r, r-2+h(X)\}$.

Intuitively, if $h$ is a localized fractional clique then $h(X)>0$ implies that "most" of the weight of $h$ is assigned to the neighbours of $X$. In particular, if $h(X) \geq 2$, then all the weight of $h$ is assigned to the neighbours of $X$. So a localized fractional clique is quite similar to a clique (in some sense). In this section, we prove that localized fractional cliques are persistent.

THEOREM 4.2. If $h$ is a localized fractional clique of a graph $H$, then $h$ is persistent.

Proof. Assume Theorem 4.2 is not true. Let $G$ be a counterexample, i.e., $G$ has a fractional clique $g$ such that

$$
\phi_{g, h}(U)>\max \left\{\omega_{f}(G), \omega_{f}(H)\right\} .
$$

Without loss of generality, we may assume that $G$ is a smallest counterexample. We shall derive a contradiction. We divide the argument into a few lemmas.

Lemma 4.3. Suppose $U$ is an independent set of $G \times H$. If $x, x^{\prime}$ are two vertices of G for which $h\left(U_{x}\right)+h\left(U_{x^{\prime}}\right)>2$ then $x \ngtr x^{\prime}$.

Proof. Assume $h\left(U_{x}\right)+h\left(U_{x^{\prime}}\right)>2$. Since $h$ is a localized fractional clique, $N\left(U_{x}\right) \cap U_{x^{\prime}} \neq \emptyset$. Let $a \in U_{x}, b \in U_{x^{\prime}}$ such that $a \sim b$. If $x \sim x^{\prime}$, then $(x, a) \sim\left(x^{\prime}, b\right)$, contrary to the assumption that $U$ is an independent set of $G \times H$. 
Corollary 4.4. Suppose $U$ is an independent set of $G \times H$. If $h\left(U_{x}\right) \geq 2$ for some vertex $x \in V(G)$, then $\phi_{g, h}(U) \leq \max \left\{\omega_{f}(G), \omega_{f}(H)\right\}$.

Proof. Let $Y=\left\{x \in V(G): h\left(U_{x}\right) \geq 2\right\} \neq \emptyset$. By Lemma 4.3, $Y$ is an independent set of $G$, and for any $x \in N(Y), f\left(U_{x}\right)=0$.

Let

$$
U^{\prime}=U \cap(V(G)-N[Y]) \times V(H) .
$$

Then $U^{\prime}$ is an independent set of $(G-N[Y]) \times H$. Let $g^{\prime}: V(G)-N[Y] \rightarrow[0,1]$ be the mapping defined as

$$
g^{\prime}(x)=\frac{g(x)}{(1-g(Y))}
$$

for any $x \in V(G)-N[Y]$. Since for any independent set $Z$ of $G-N[Y], Y \cup Z$ is an independent set of $G$, hence $g(Y)+g(Z) \leq 1$, which implies that

$$
g^{\prime}(Z)=g(Z) /(1-g(Y)) \leq 1 .
$$

Hence $g^{\prime}$ is a fractional clique of $G-N[Y]$.

By the minimality of $G$, we have

$$
\begin{aligned}
\phi_{g^{\prime}, h}\left(U^{\prime}\right)=\sum_{(x, a) \in U^{\prime}} g^{\prime}(x) h(a) & \leq \max \left\{\omega_{f}(G-N[Y]), \omega_{f}(H)\right\} \\
& \leq \max \left\{\omega_{f}(G), \omega_{f}(H)\right\}
\end{aligned}
$$

As $g(x)=(1-g(Y)) g^{\prime}(x)$, we have

$$
\sum_{(x, a) \in U^{\prime}} g(x) h(a) \leq(1-g(Y)) \max \left\{\omega_{f}(G), \omega_{f}(H)\right\} .
$$

Hence

$$
\begin{aligned}
\phi_{g, h}(U) & =\sum_{(x, a) \in U} g(x) h(a) \\
& \leq \sum_{x \in Y} g(x) h(H)+\sum_{(x, a) \in U^{\prime}} g(x) h(a) \\
& \leq g(Y) h(H)+(1-g(Y)) \max \left\{\omega_{f}(G), \omega_{f}(H)\right\} \\
& \leq \max \left\{\omega_{f}(G), \omega_{f}(H)\right\} .
\end{aligned}
$$

Let $U$ be an independent set of $G \times H$ such that $\phi_{g, h}(U)>\max \left\{\omega_{f}(G), \omega_{f}(H)\right\}$. Let $t=\max \left\{h\left(U_{x}\right): x \in V(G)\right\}$. By Corollary 4.4, $t<2$. If $t \leq 1$, then

$$
\begin{aligned}
\sum_{(x, a) \in U} g(x) h(a) & =\sum_{x \in V(G)} g(x) h\left(U_{x}\right) \\
& \leq \sum_{x \in V(G)} g(x) \\
& \leq \max \left\{\omega_{f}(G), \omega_{f}(H)\right\},
\end{aligned}
$$


contrary to our assumption. Thus $1<t<2$. For any $0<s \leq t$, let

$$
Y_{s}=\left\{x \in V(G): h\left(U_{x}\right)=s\right\} .
$$

Since $G$ is finite, there are only finitely many $s$ for which $Y_{s} \neq \emptyset$. Let

$$
s_{0}>s_{1}>s_{2}>\cdots>s_{m}
$$

be the real numbers such that $2>s_{i}>1$ and either $Y_{s_{i}} \neq \emptyset$ or $Y_{2-s_{i}} \neq \emptyset$.

For $i=0,1,2, \cdots, m$, let

$$
Z_{s_{i}}=\left\{x \in V(G): h\left(U_{x}\right)=2-s_{i}\right\}
$$

and let

$$
B=\left\{x \in V(G): h\left(U_{x}\right)=1\right\} .
$$

As $t<2$, by definition, for any $x \notin B \cup\left(\cup_{i=0}^{m}\left(Y_{s_{i}} \cup Z_{s_{i}}\right)\right), \quad h\left(U_{x}\right)=0$. Hence $\phi(U)=g(B)+\sum_{i=0}^{m}\left(g\left(Y_{s_{i}}\right) s_{i}+g\left(Z_{s_{i}}\right)\left(2-s_{i}\right)\right)$.

We shall construct a new independent set $U^{\prime}$ of $G \times H$ such that $\phi_{g, h}\left(U^{\prime}\right) \geq$ $\phi_{g, h}(U)$, and $h\left(U_{x}^{\prime}\right) \geq 2$ for some $x \in V(G)$. But this is a contradiction, because then it would follow from Corollary 4.4 that $\phi_{g, h}\left(U^{\prime}\right) \leq \max \left\{\omega_{f}(G), \omega_{f}(H)\right\}$.

The independent set $U^{\prime}$ is obtained from $U$ by shifting some elements from $U_{x}$ to $U_{y}$ for some $x$ and $y$. First we need a technical lemma.

Lemma 4.5. Suppose $\alpha_{0}, \alpha_{1}, \cdots, \alpha_{m}$ and $\beta_{0}, \beta_{1}, \cdots, \beta_{m}$ are positive real numbers such that $\frac{\beta_{i}}{\alpha_{i}} \geq \frac{\beta_{i+1}}{\alpha_{i+1}}$ for $i=0,1, \cdots, m-1$. If $x_{0}, x_{1}, \cdots, x_{m}$ is a sequence of reals satisfying $\sum_{j=0}^{i} \alpha_{j} x_{j}>0$ for all $0 \leq i \leq m$, then $\sum_{j=0}^{i} \beta_{j} x_{j}>0$ for all $0 \leq i \leq m$.

Proof. Suppose $\sum_{j=0}^{i} \alpha_{j} x_{j}>0$ for all $0 \leq i \leq m$. Let $0 \leq s \leq m$. Then

$$
\begin{aligned}
& \frac{\beta_{s}}{\alpha_{s}} \sum_{j=0}^{s} \alpha_{j} x_{j}>0, \\
&\left(\frac{\beta_{s-1}}{\alpha_{s-1}}-\frac{\beta_{s}}{\alpha_{s}}\right) \sum_{j=0}^{s-1} \alpha_{j} x_{j} \geq 0, \\
&\left(\frac{\beta_{s-2}}{\alpha_{s-2}}-\frac{\beta_{s-1}}{\alpha_{s-1}}\right) \sum_{j=0}^{s-2} \alpha_{j} x_{j} \geq 0, \\
&\left(\frac{\beta_{1}}{\alpha_{1}}-\frac{\beta_{2}}{\alpha_{2}}\right) \sum_{j=0}^{1} \alpha_{j} x_{j} \geq 0, \\
&\left(\frac{\beta_{0}}{\alpha_{0}}-\frac{\beta_{1}}{\alpha_{1}}\right) \alpha_{0} x_{0} \geq 0 .
\end{aligned}
$$

The summation of both sides of the above inequalities gives the inequality

$$
\sum_{j=0}^{s} \beta_{j} x_{j}>0 .
$$


Now we start to construct $U^{\prime}$. For $i=0,1, \cdots, m$, let $x_{i}=g\left(Z_{s_{i}}\right)-g\left(Y_{s_{i}}\right)$, $\alpha_{i}=2-s_{i}$ and $\beta_{i}=s_{i}-1$. Note that

$$
\alpha_{0}<\alpha_{1}<\cdots \alpha_{m}
$$

and

$$
\beta_{0}>\beta_{1}>\cdots>\beta_{m}
$$

Hence

$$
\frac{\beta_{i}}{\alpha_{i}}>\frac{\beta_{i+1}}{\alpha_{i+1}}
$$

for $i=0,1, \cdots, m-1$.

If $\sum_{j=0}^{i} \alpha_{i} x_{i}>0$ for all $0 \leq i \leq m$, then, by Lemma $4.5, \sum_{j=0}^{i} \beta_{i} x_{i}>0$ for all $0 \leq i \leq m$. Hence

$$
\begin{aligned}
\phi_{g, h}(U) & =\sum_{(x, a) \in U} g(x) h(a) \\
& =\sum_{x \in V(G)} g(x) h\left(U_{x}\right) \\
& =g(B)+\sum_{j=0}^{m}\left(g\left(Y_{s_{j}}\right) s_{j}+g\left(Z_{s_{j}}\right)\left(2-s_{j}\right)\right) \\
& =g(B)+\sum_{j=0}^{m}\left(g\left(Y_{s_{j}}\right)+g\left(Z_{s_{j}}\right)\right)-\sum_{j=0}^{m}\left(g\left(Z_{s_{j}}\right)-g\left(Y_{s_{j}}\right)\right)\left(s_{j}-1\right) \\
& =g(B)+\sum_{j=0}^{m}\left(g\left(Y_{s_{j}}\right)+g\left(Z_{s_{j}}\right)\right)-\sum_{j=0}^{m} \beta_{j} x_{j} \\
& <g(B)+\sum_{j=0}^{m}\left(g\left(Y_{s_{j}}\right)+g\left(Z_{s_{j}}\right)\right) \\
& \leq g(V(G)) \\
& \leq \max \left\{\omega_{f}(G), \omega_{f}(H)\right\} .
\end{aligned}
$$

Thus we may assume that there exists $0 \leq i \leq m$ such that $\sum_{j=0}^{i} \alpha_{j} x_{j} \leq 0$. Let $U^{\prime}$ be the independent set of $G \times H$ defined as

- $U_{x}^{\prime}=V(H)$ if $x \in Y_{s_{j}}$ for some $j \leq i$;

- $U_{x}^{\prime}=\emptyset$ if $x \in Z_{s_{j}}$ for some $j \leq i$;

- $U_{x}^{\prime}=U_{x}$ otherwise.

By Lemma 4.3, $U^{\prime}$ is indeed an independent set of $G \times H$. On the other hand, 


$$
\begin{aligned}
\phi_{g, h}\left(U^{\prime}\right) & =\sum_{(x, a) \in U^{\prime}} g(x) h(a) \\
& =\sum_{(x, a) \in U} g(x) h(a)-\sum_{x \in \bigcup_{j=0}^{i} Z_{s_{j}},(x, a) \in U} g(x) h(a)+\sum_{\substack{i \\
j=0}} g(x) h(a) \\
& =\sum_{(x, a) \in U} g(x) h(a)-\sum_{j=0}^{i} g\left(Z_{s_{j}},(x, a) \notin U\right. \\
& \geq \sum_{(x, a) \in U} g(x) h(a)-\sum_{j=0}^{i} \alpha_{j} x_{j} \\
& \geq \phi_{g, h}(U) .
\end{aligned}
$$

Since $\max \left\{f\left(U_{x}^{\prime}\right): x \in V(G)\right\} \geq 2$, by Corollary 4.4, we have

$$
\phi_{g, h}\left(U^{\prime}\right) \leq \max \left\{\omega_{f}(G), \omega_{f}(H)\right\}
$$

This proves Theorem 4.2.

5. The graphs $G_{d}^{k}$ and Kneser graphs. As a consequence of Theorem 4.2, we have the following sufficient conditions for a graph to be fractional persistent and strongly fractional persistent.

COROLlary 5.1. If a graph $H$ has a localized fractional clique $h$ of weight $w(h)=\omega_{f}(H)$ then $H$ is fractional persistent; if all the fractional cliques of $H$ are localized fractional cliques then $H$ is strongly fractional persistent.

This section proves that an important class of graphs, the graphs $G_{d}^{k}$, satisfy the conditions of Corollary 5.1, and hence are strongly fractional persistent.

Suppose $k \geq 2 d$ are positive integers. The graph $G_{d}^{k}$ has vertices $0,1, \cdots, k-1$, and $i \sim j$ if and only if $d \leq|i-j| \leq k-d$. The vertices of $G_{d}^{k}$ are usually viewed as forming a circle $C^{k}$. We denote by $[i, j]_{k}$ the set $\{i, i+1, \cdots, j\}$, where arithmetic is modulo $k$. For example, $[2,5]_{k}=\{2,3,4,5\}$ and $[5,2]_{k}=\{5,6, \cdots, k-1,0,1,2\}$. The addition and subtraction on the vertices of $G_{d}^{k}$ are always carried out modulo $k$.

The graphs $G_{d}^{k}$ are special circulant graphs which play an important role in the study of circular chromatic number of graphs [14].

THEOREM 5.2. Suppose $f$ is a fractional clique of $G_{d}^{k}$ of weight $r$ and $X$ is a subset of $V\left(G_{d}^{k}\right)$. If $f(X)=t$ for some $0<t \leq 2$, then $f(N(X)) \geq r-2+t$. If $f(X) \geq 2$ then $f(N(X))=r$.

Proof. We say a subset $X$ of $V\left(G_{d}^{k}\right)$ is critical if for any proper superset $X^{\prime} \supset X$, $N\left(X^{\prime}\right)$ is a proper superset of $N(X)$. It is obvious that it suffices to prove Lemma 5.2 for critical subsets $X$ of $V\left(G_{d}^{k}\right)$. So in the following, we assume that $X$ is a critical subset of $V\left(G_{d}^{k}\right)$. 
First we prove this lemma for the case that $X=[a, b]_{k}$ is an interval of $C^{k}$. If the length of the interval is at least $2 d$, then it follows from the definition that $N(X)=V\left(G_{d}^{k}\right)$, and we are done. Assume the length of the interval is $q \leq 2 d-1$. Then $N(X)=[a+d, b-d]_{k}$. So

$$
f(N(X))=r-f\left([b-d+1, a+d-1]_{k}\right) .
$$

Let $A=[b-d+1, b]_{k}$ and $B=[a, a+d-1]_{k}$. Then both $A$ and $B$ are independent sets, so $f(A)+f(B) \leq 2$. Hence

$$
f\left([b-d+1, a+d-1]_{k}\right)=f(A)+f(B)-f\left([a, b]_{k}\right) \leq 2-t .
$$

So $f(N(X)) \geq r-2+t$.

Assume now that $X$ is not an interval, say

$$
X=\left[a_{1}, b_{1}\right]_{k} \cup\left[a_{2}, b_{2}\right]_{k} \cup \cdots \cup\left[a_{s}, b_{s}\right]_{k},
$$

where $\left[a_{i}, b_{i}\right]$ are the maximal intervals contained in $X$. Let $X_{i}=\left[a_{i}, b_{i}\right]_{k}$. Observe that $N\left(X_{i}\right)=\left[a_{i}+d, b_{i}-d\right]_{k}$ is also an interval of $C^{k}$. If there are two intervals $X_{i}, X_{j}$ such that $N\left(X_{i}\right) \cap N\left(X_{j}\right) \neq \emptyset$, then $N\left(X_{i}\right) \cup N\left(X_{j}\right)$ is an interval, and it is easy to see that $N\left(X_{i}\right) \cup N\left(X_{j}\right)=N\left(\left[a_{i}, b_{j}\right]_{k}\right)$ or $N\left(X_{i}\right) \cup N\left(X_{j}\right)=N\left(\left[a_{j}, b_{i}\right]_{k}\right)$. This is contrary to the assumption that $X$ is a critical subset of $V\left(G_{d}^{k}\right)$. Thus we may assume that $N\left(X_{i}\right) \cap N\left(X_{j}\right)=\emptyset$ for all $i \neq j$. Hence $f(N(X))=\sum_{i=1}^{s} f\left(N\left(X_{i}\right)\right)$. As $X_{i}$ is an interval, so we have $f\left(N\left(X_{i}\right)\right) \geq r-2+f\left(X_{i}\right)$. Therefore

$$
f(N(X)) \geq \sum_{i=1}^{s} r-2+f\left(X_{i}\right)=(r-2) s+t \geq r-2+t .
$$

COROllary 5.3. If $k_{i} \geq 2 d_{i}$ for $i=1,2, \cdots, t$ and $G$ is the direct sum of $G_{d_{i}}^{k_{i}}$ $(i=1,2, \cdots, t)$, then $G$ is strongly fractional persistent. Hence, for any graph $H$,

$$
\chi_{f}(G \times H)=\min \left\{\chi_{f}(G), \chi_{f}(H)\right\} .
$$

Suppose $k \geq 2 d$. The Kneser graph $K(k, d)$ has as vertices all the $d$-subsets of $\{0,1, \cdots, k-1\}$, and two vertices are adjacent if and only if the two subsets are disjoint. Kneser graphs play a special role in the study of fractional chromatic numbers of graphs, and have been studied extensively in the literature (see [9]).

A graph $G$ has a fractional colouring of weight $k / d$ if and only if $G$ admits a homomorphism to $K(l k, l d)$ for some positive integer $l$. So, for fractional colouring, the Kneser graphs play the role of complete graphs in the study of ordinary colouring, and the role of $G_{d}^{k}$ in the study of circular colouring.

THeOrem 5.4. If $k_{i} \geq 2 d_{i}$ for $i=1,2, \cdots, t$ and $H$ is the direct sum of $K\left(k_{i}, d_{i}\right)$ $(i=1,2, \cdots, t)$, then $H$ is fractional persistent.

Proof. Let $H^{\prime}$ be the direct sum of $G_{d_{i}}^{k_{i}}(i=1,2, \cdots, t)$. It is easy to verify that

$$
\chi_{f}\left(H^{\prime}\right)=\sum_{i=1}^{t} \frac{k_{i}}{d_{i}}=\chi_{f}(H)
$$


On the other hand, $G_{d}^{k}$ admits a homomorphism to $K(k, d)$ for any $k \geq 2 d$ (the mapping sending $i$ to $\{i, i+1, \cdots, i+d-1\}$ is a homomorphism from $G_{d}^{k}$ to $K(k, d)$ ). Therefore $H^{\prime}$ admits a homomorphism to $H$. As $H^{\prime}$ is fractional persistent, by Lemma 3.3, $H$ is fractional persistent.

For a graph $G$, we denote by $\alpha(G)$ the independence number of $G$ which is defined as the order of a maximum independent set of $G$. It is well-known [9] that for a vertex-transitive graph $G$ we have $\chi_{f}(G)=|V(G)| / \alpha(G)$. Thus we have the following corollary:

COROLlary 5.5. Suppose $G$ is a vertex-transitive graph and $H$ is either a circulant graph $G_{d}^{k}$ (for some $k \geq 2 d$ ), or a Kneser graph $K(k, d)$ (for some $k \geq 2 d$ ). Then

$$
\alpha(G \times H)=\max \{\alpha(G)|V(H)|, \alpha(H)|V(G)|\} .
$$

The following special case of Corollary 5.5 is a generalization of the Erdős-KoRado theorem, and was proved in [3]:

Corollary 5.6. Suppose $k_{i}, d_{i}$ are positive integers and $k_{1} / d_{1} \geq k_{2} / d_{2} \geq \cdots \geq$ $k_{t} / d_{t} \geq 2$. Then

$$
\alpha\left(K\left(k_{1}, d_{1}\right) \times K\left(k_{2}, d_{2}\right) \times \cdots \times K\left(k_{t}, d_{t}\right)\right)=\left(\begin{array}{l}
k_{1}-1 \\
d_{1}-1
\end{array}\right)\left(\begin{array}{l}
k_{2} \\
d_{2}
\end{array}\right)\left(\begin{array}{l}
k_{3} \\
d_{3}
\end{array}\right) \cdots\left(\begin{array}{l}
k_{t} \\
d_{t}
\end{array}\right) .
$$

\section{Some remarks.}

(1) We have shown a natural class of graphs $G$ which are strongly fractional persistent, by showing that all the fractional cliques of $G$ are localized fractional cliques. Certainly most graphs have fractional cliques which are not localized. For example, for most graphs $G_{d}^{k}$, their complements have non-localized fractional cliques. It would be interesting to find new methods to prove that such graphs are (strongly) fractional persistent.

(2) It is known [10] that for some Kneser graphs $K(k, d)$, there exist graphs $G$ and $H$ such that $G \times H$ admits a homomorphism to $K(k, d)$ and yet none of $G, H$ admits a homomorphism to $K(k, d)$. However, this does not provide a negative answer to Question 1.1, as $G$ or $H$ may still has fractional chromatic number at most $k / d$. Actually, this is the case for the examples given in [10].

(3) The fractional chromatic numbers of other product graphs have also been studied in the literature. There is a simple formula for the fractional chromatic number of the lexicographic product $G[H]$ of $G$ and $H: \chi_{f}(G[H])=\chi_{f}(G) \chi_{f}(H)$ [2]. This formula is also true for the strong product $G \otimes H$ (where $\left(x, x^{\prime}\right) \sim\left(y, y^{\prime}\right)$ if and only if $x \sim x^{\prime}$ or $\left.y \sim y^{\prime}\right)$ of $G$ and $H: \chi_{f}(G \otimes H)=\chi_{f}(G) \chi_{f}(H)$ [9]. For the Cartesian product $G \square H$ (where $\left(x, x^{\prime}\right) \sim\left(y, y^{\prime}\right)$ if and only if either $x \sim x^{\prime}$ and $y=y^{\prime}$ or $x=x^{\prime}$ and $y \sim y^{\prime}$ ), the fractional chromatic number of $G \square H$ is difficult to determine. Even for the powers of a single graph, many question concerning the fractional chromatic number remain open. For example, let $G^{n}=G^{n-1} \square G$ $\left(G^{1}=G\right)$, then it is unknown if the $\operatorname{limit}_{n \rightarrow \infty} \chi_{f}\left(G^{n}\right)$ is a rational number. This problem is related to the ultimate independence ratio of a graph, which was studied in $[6,13]$. 
AcKnowledgements. The author benefits from discussions with S. Klav̌zar, C. Tardif and H. Yeh. He also thanks the referee for insightful comments.

\section{REFERENCES}

1. H. El-Zahar and N. Sauer, The chromatic number of the product of two 4-chromatic graphs is 4, Combinatorica 5 (1985), 121-126.

2. G. Gao and X. Zhu, Star-extremal graphs and the lexicographic product, Discrete Mathematics 152 (1996), 147-156.

3. P. Frankl, An Erdős-Ko-Rado theorem for direct products, European J. Combin. 17 (1996), 727-730.

4. R. Häggkvist, P. Hell, D.J. Miller and V. Neumann-Lara, On multiplicative graphs and the product conjecture, Combinatorica 8 (1988), 71-81.

5. S. H. Hedetniemi, Homomorphisms of graphs and automata, University of Michigan Technical Report 03105-44-T (1966).

6. $\mathrm{P}, \mathrm{Hell}, \mathrm{X}$. $\mathrm{Yu}$ and $\mathrm{H}$. Zhou, Independence ratio of graph powers, Discrete Mathematics 127 (1994), 213-220.

7. P. Jha and S. Klavžar, Independence in direct-product graphs, Ars Combinatoria 50 (1998), 53-63.

8. Y. Kirsch, Bounding the independence number via graph product, manuscript.

9. E. R. Scheinerman and D. H. Ullman, Fractional graph theory, Wiley-Interscience Series in Discrete Mathematics and Optimization (John Wiley \& Sons, New York, 1997).

10. C. Tardif and X. Zhu, The level of nonmultiplicativity of graphs, Discrete Math., to appear.

11. N. Sauer and X. Zhu, An approach to Hedetniemi's conjecture, J. Graph Theory $\mathbf{1 6}$ (1992), 423-436.

12. C. Tardif, Graph products and the chromatic difference sequence of vertex-transitive graphs, Discrete Math. 185 (1998), 193-200.

13. X. Zhu, On the bound for the ultimate independence ratio of a graph, Discrete Math. 156 (1996), 229-236.

14. X. Zhu, Circular chromatic number, a survey, Discrete Math., 229 (2001), 371-410.

15. X. Zhu, A survey on Hedetniemi's conjecture, Taiwanese J. Math 2 (1998), 1-24. 